\title{
Effectiveness of Greater Occipital Nerve Blocks in Migraine Prophylaxis
}

\author{
Nurten iNAN', Levent E. iNAN², Özlem COŞKUN³, Tuğba TUNÇ4, Mustafa iLHAN5 \\ 'Department of Anaesthesiology and Algology, Gazi University School of Medicine, Ankara, Turkey \\ ${ }^{2}$ Neurology and Algology, Ministry of Health Ankara Training and Research Hospital, Ankara, Turkey \\ 3 Department of Neurology and Algology, Gazi University School of Medicine, Ankara, Turkey \\ ${ }^{4}$ Department of Neurology, Gazi University School of Medicine, Ankara, Turkey \\ ${ }^{5}$ Department of Public Health, Gazi University School of Medicine, Ankara, Turkey
}

\section{ABSTRACT}

Introduction: Peripheral nerve blocks have been used in primary headache treatment since a long time. In this study, we aimed to examine the efficiency of greater occipital nerve (GON) block in migraine prophylaxis.

Methods: Data from migraine without aura patients who had GON block were collected and divided into two groups: Group PGON $(n=25)$, which included patients who were under medical prophylaxis and had GON block, and Group GON ( $=53)$, which included patients who had only GON blocks. Migraine was diagnosed using International Headache Society (IHS) classification. Data of 78 patients were analyzed. Headache attack frequency, headache duration, and severity were compared between and within groups in a 3-month follow-up period.

Results: The decrease in headache parameters after GON block in both groups was significantly similar. Headache attack frequency decreased from 15.73 \pm 7.21 (pretreatment) to $4.52 \pm 3.6$ I (3rd month) in Group GON and from $13.76 \pm 8.07$ to $3.28 \pm 2.15$ in Group PGON $(p<0.05)$.
Headache duration decreased from $18.51 \pm 9.43$ to $8.02 \pm 5.58$ at 3 rd month in Group GON and from 15.20 \pm 9.16 to $7.20 \pm 4.16$ in Group PGON $(p<0.05)$. Headache severity decreased from $8.26 \pm 1.32$ to $5.16 \pm 2.64$ in Group GON and from $8.08 \pm 0.90$ to $5.96 \pm 1.20$ in Group PGON $(p<0.05)$. There was no statistically significant difference between the groups in 3rd month after treatment $(p>0.05)$

Conclusion: This study showed significant decreases in headache parameters in both groups. As GON blocks were performed in patients unresponsive to medical prophylaxis, a decrease in the headache parameters in Group PGON similar to that in Group GON can be attributed to GON blocks. Consequently, these results show that repeated GON blocks with local anesthetic can be an effective alternative treatment in migraine patients who are unresponsive to medical prophylaxis or who do not prefer to use medical prophylaxis.

Keywords: Headache, migraine, prophylaxis, greater occipital nerve block

\section{INTRODUCTION}

Migraine is a common disorder, and its prevalence in Turkey has reached I6\% (I). Prophylactic treatment with antidepressants, antiepileptics, calcium channel blockers, and beta blockers may help in reducing attack frequency, severity, and duration (2). Some patients may not respond to prophylactic agents. In the literature, greater occipital nerve (GON) blocks with local anesthetics and steroids were used in migraine patients, and some benefits in acute migraine and prophylaxis were obtained $(3,4,5,6)$.

In this study, our aim was to test the effectiveness of GON blocks in migraine prophylaxis in patients who used other prophylactic agents and to compare the results with those of the patients who used only GON blocks without other prophylactic agents.

To our knowledge, this is the first study to compare the effectiveness of GON block in patients under medical prophylaxis with that of GON block alone in migraine patients.

\section{METHODS}

After approval from the ethics committee of Gazi University, this retrospective study was conducted in Algology and Headache departments between 2012 and 2014 in migraine without aura patients. Classification of the headache diagnosis as of the date that a patient was included in the study was made according to the diagnosis criteria of the International Headache Society published in 2004 (7). Patients who had maintained a headache diary for I month after the diagnosis of migraine without aura and for 3 months during GON block treatment were included in the study.

The exclusion criteria were as follows: previous occipital nerve block or occipital nerve stimulation, a history of occipital region surgery, a history of allergic reaction to the local anesthetic to be applied, pregnancy and breastfeeding, existing active psychiatric disease, uncontrolled

Correspondence Address: Nurten İnan, Gazi Üniversitesi Tıp Fakültesi, Anesteziyoloji ve Reanimasyon Anabilim Dalı, Algoloji Bilim Dalı, Ankara, Türkiye E-mail: nurteninan@yahoo.com

Received: 10.10.2014 Accepted: 17.12.2014

(C) Copyright 2016 by Turkish Association of Neuropsychiatry - Available online at www.noropskiyatriarsivi.com 
hypertension, uncontrolled diabetes mellitus, decompensated congestive heart failure, chronic renal failure, chronic liver disease, tumor and/ or the presence of vascular disease, any existing inflammatory and/or infectious disease, and the use of anticoagulants.

In all patients, the GON blockades were performed in the Algology department by the same algologist. The patients were divided into two groups: Group PGON ( $n=25)$ and Group GON ( $n=53)$. The former included patients who took at least 3 months or more for medical prophylaxis (amitriptyline $25 \mathrm{mg} /$ day, topiramate $100 \mathrm{mg} /$ day, or venlafaxine 150/day) but did not respond efficiently and were started on GON blockade and the latter included patients who only underwent GON blockade. Patients who were in Group PGON had a history of different medical prophylaxis treatment.

In Group PGON and Group GON, GON blocks were repeated every week in the first month $\left(\mathrm{I}^{\text {st }}, 2^{\text {nd }}, 3^{\text {rd }}, 4^{\text {th }}\right.$ week $)$ and repeated monthly for the following 2 months. During these periods, patients in both groups maintained a diary to record headache duration (hours), headache frequency (days), and severity of pain (visual analog scale, VAS). The injections were performed radially at $2 \mathrm{~cm}$ lateral and $2 \mathrm{~cm}$ inferior to the protuberantia occipitalis externa with a $26 \mathrm{G} 0.45 \times 13 \mathrm{G}$ needle and $2 \mathrm{~mL}$ of $0.25 \%$ bupivacaine (Figure I). The patients were kept under observation for 30 minutes to note the possible side effects.

Headache severity was recorded using a VAS. All patients were informed about the scale numbered from 0 (no pain) to 10 (worst pain) and were asked to indicate pain severity with VAS in the headache diary. The severity of pain (VAS), pain frequency, and pain duration were compared between the two groups and between the groups before (values in the last month) and after the blocks.

\section{Statistical Analysis}

The results were compared using Statistical Package for the Social Sciences (SPSS Inc.; Chicago, IL, USA) I 5.0 for Windows. Student's t-tests were used for continuous variables with a normal distribution for the mean comparisons between the groups. The chi-square test and Fisher's exact test were used for comparison of variables distributed in percentage. Repeated measures analysis of variance (ANOVA) and paired sample t-tests were used for variables with a normal distribution for the comparisons within groups. A value of $p<0.05$ was considered to be significant in the two-tailed analysis.

\section{RESULTS}

Demographic data and the characteristics of pain are shown in Table I. Seventy-eight patients were included into the study. The data of 53 patients in Group GON and 25 in Group PGON were included in the study. The pretreatment headache frequencies in Group PGON and in Group GON were statistically similar (Table 2). In Group PGON and Group $\mathrm{GON}$, the headache attack frequencies were significantly decreased in the $1^{\text {st }}, 2^{\text {nd }}$, and $3^{\text {rd }}$ months after GON block from those in the period before the block $(p<0.00 I)$ (Table 2). There were no significant differences for this parameter between the groups $(p>0.05)$, except in the $1^{\text {st }}$ week.
The pretreatment headache duration values were also similar in both groups. When a comparison was made in the duration of headache between Group PGON and Group GON, there was a statistically significant decrease in the values for the $1^{\text {st }}, 2^{\text {nd }}, 3^{\text {rd }}$, and $4^{\text {th }}$ weeks as well as for the $2^{\text {nd }}$ and $3^{\text {rd }}$ months from those recorded before the block period $(p<0.00$ I, Table 3$)$. There were significant differences between the groups in terms of headache duration only in the $1^{\text {st }}, 2^{\text {nd }}$, and $4^{\text {th }}$ weeks; the headache durations of Group PGON were significantly lower in this follow-up period ( $p<0.05$, Table 3 ).

The pretreatment headache severity values were similar in both groups. In Group PGON and Group GON, the mean pain severities (VAS) decreased in a statistically significant manner in the $1^{\text {st }}, 2^{\text {nd }}, 3^{\text {rd }}$, and $4^{\text {th }}$ weeks and in the $2^{\text {nd }}$ and $3^{\text {rd }}$ months from those values before the blo$c k(p<0.00$ I, Table 4). There were no significant differences in severity between the groups ( $p>0.05$, Table 4$)$.

\section{DISCUSSION}

This study showed that repetitive GON blocks with bupivacaine decreased the number of headache days, duration, and VAS scores significantly in mig-

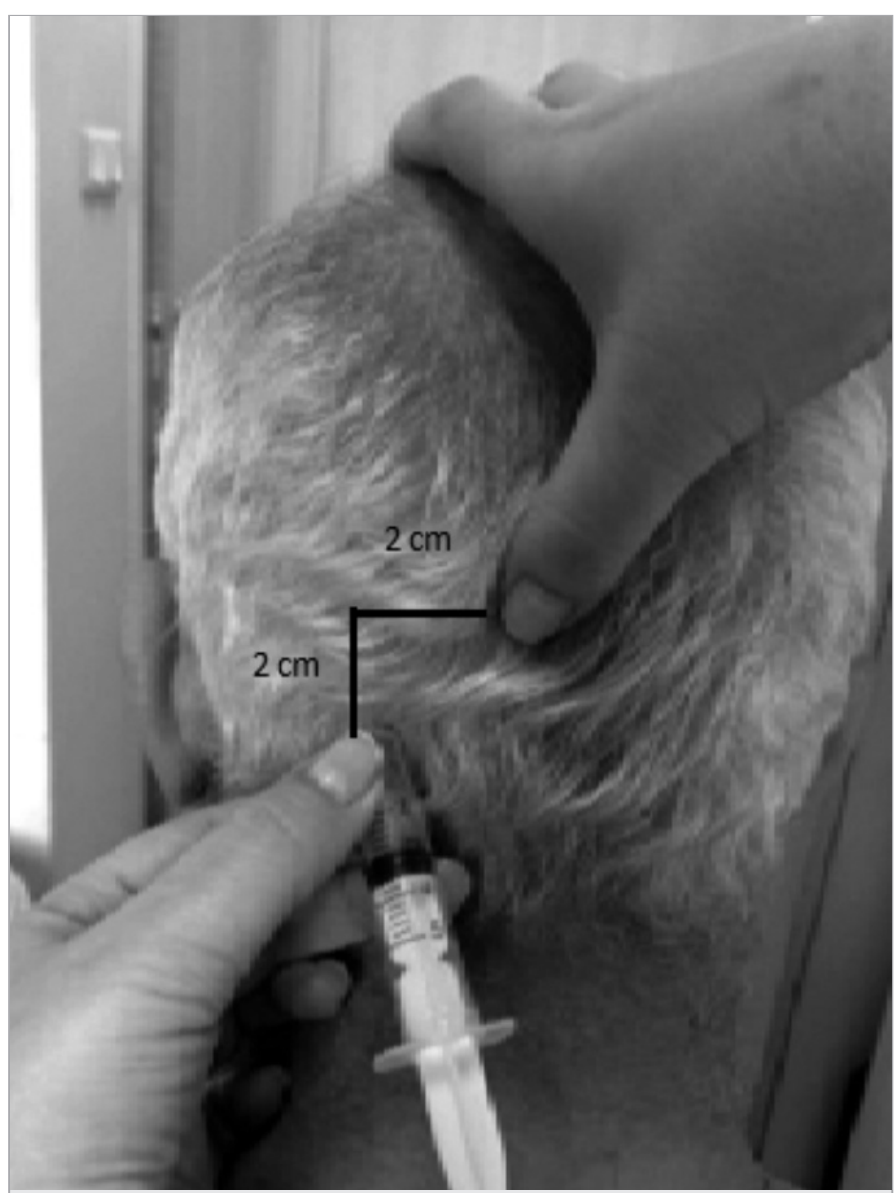

Figure I. Greater occipital nerve block; the entry point is $2 \mathrm{~cm}$ at the lateral and $2 \mathrm{~cm}$ distal from external occipital protuberance

Table I. Comparison of demographic data and characteristics of headache.

\begin{tabular}{|c|c|c|c|c|}
\hline & \multicolumn{2}{|c|}{ Group GON $(n=53)$} & \multicolumn{2}{|c|}{ Group PGON (n=25) } \\
\hline Age (years) (mean $\pm S D)$ & \multicolumn{2}{|c|}{$37.83 \pm 8.73$} & \multicolumn{2}{|c|}{$40.08 \pm 9.74$} \\
\hline Side of pain (right/left/bilateral) (n/\%) & $|8 /| 4 / 2 \mid$ & $34 \% / 26.4 \% / 39.6 \%$ & $1 \mid / 5 / 9$ & $44 \% / 20 \% / 36 \%$ \\
\hline Female/male (n/\%) & $49 / 4$ & $92.5 \% / 7.5 \%$ & $19 / 6$ & $76 \% / 24 \%$ \\
\hline
\end{tabular}


raine patients. Patients who were unresponsive to oral prophylactic agents and patients who had not taken medical prophylaxis gave similar responses. The headache duration was decreased more with GON block in patients under medical prophylaxis in the $1^{\text {st }}, 2^{\text {nd }}$, and $4^{\text {th }}$ weeks than in patients with GON block alone, but this duration became equal in both groups at the other follow-up periods, and the headache frequency was decreased more in Group PGON than in Group GON only in the $1^{\text {st }}$ week.

In the present study, all patients (with or without medical prophylaxis) had similar results in terms of headache days, duration, and severity in the last month before treatment; therefore, this is probably the reason for the similar results of both groups after 3 months of treatment.

The effectiveness of GON blockade in migraine has been shown in several studies $(8,9,10,11,12,13,14,15)$. The results of the present study suggest that prophylactic drug treatment in migraine patients did not increase the benefits of GON block in a 3-month follow-up period. In other words, GON block does not have a summation effect on prophylactic drugs in 3-month follow-up periods in migraine patients, and we found that GON blocks were effective in the treatment of all migraine patients in both groups.

Blumenfeld et al. (16) investigated the use of peripheral nerve blocks and trigger point injections in headache patients by headache practitioners in the USA. They found that $69 \%$ of responders used peripheral nerve blo$\mathrm{cks}$, and the most common indication was occipital neuralgia and chronic migraine. Despite the worldwide usage of nerve blocks in the treatment of headaches, there are limited evidence-based studies within the literature. The combination of GON blocks with medical prophylaxis had not been studied before. For this reason, the results of the present study are important for conducting future research.

Similar to the results of the study by Blumenfeld et al. (16), in the present study, no major side effects were observed after GON block. Only local pain, numbness, vertigo, and nausea were recorded. We also stated that 30 minutes of observation after GON blocks is important to prevent major side effects.

The efficacy of GON blocks in the treatment of various types of headache has been assessed in several studies (17,18,19,20,21). Decreases in headache parameters with GON blocks exceed the half-life of local anesthetics. The observed response was probably attributable to factors other than the direct local anesthetic effect of the GON block $(22,23)$. Changes or modulation in brain nociceptive pathways may have a role in this effect. Another possible explanation for these findings is that the injections themselves initiated diffuse noxious inhibitory control, independent of the anesthetic effect (I0). Functional connectivity between the sensory occipital segments and the trigeminal nociceptive system in humans may also play a role. The trigeminocervical complex is connected to the nucleus salivatorius with the raphe nucleus, the locus coeruleus, and consequently with the hypothalamus. In addition, painful stimuli originating from cranial structures are transmitted by means of the trigeminal nerve and superior cervical nerves to the trigeminocervical complex and then to the upper centers (23). According to recent investigations, nerve blocks are fast becoming an alternative treatment for headaches, and our results suggest that repeated GON blocks either with prophylactic agents or alone can be considered an effective management tool in migraine treatment.

In our Algology department, the GON block treatment protocol, which was based on our experience, was uniformly applied to all migraine patients. This protocol involves performing the procedure once per week in the first month, once per month for the following 2 months, and after that, depending on the patient, once every 2 to 6 months. The GON block was made with $2 \mathrm{~mL}$ of $0.25 \%$ bupivacaine without steroids. In the literature, the intervals between injections ranged from I to 8 weeks with local anesthetic and from 2 to 12 weeks with steroids (17). In the present study, injections were administered at the same intervals for all patients, independently from headache periods. With this protocol, the number of headache days decreased from 15.73 to 4.52 in group B, and from 13.76 to 3.28 in the prophylactic medication plus GON block group. While headache severity decreased from 8.26 to 5.16 and headache duration decreased from 18.51 to 8.02 in group $B$, the reduction of headache severity

Table 2. Comparison of the headache attack frequency (mean $\pm S D$ )

\begin{tabular}{|l|c|c|c|}
\hline & $\begin{array}{c}\text { Group GON } \\
(\mathbf{n = 5 3 )}\end{array}$ & $\begin{array}{c}\text { Group PGON } \\
(\mathbf{n = 2 5})\end{array}$ & $\mathbf{p}$ \\
\hline Before block & $15.73 \pm 7.21$ & $13.76 \pm 8.07$ & 0.303 \\
\hline Week I & $2.49 \pm 1.93$ & $1.52 \pm 1.12^{\#}$ & 0.007 \\
\hline Week 2 & $1.92 \pm 1.46$ & $1.64 \pm 1.18$ & 0.364 \\
\hline Week 3 & $1.86 \pm 1.42$ & $1.60 \pm 1.04$ & 0.353 \\
\hline Week 4 & $1.54 \pm 1.29$ & $1.04 \pm 0.97$ & 0.060 \\
\hline $\begin{array}{l}\text { Ist month } \\
\text { (mean of weeks I-4) }\end{array}$ & $7.83 \pm 4.78^{*}$ & $5.80 \pm 3.59 *$ & 0.064 \\
\hline $2^{\text {nd } \text { month }}$ & $5.58 \pm 4.37 *$ & $5.00 \pm 2.51 *$ & 0.458 \\
\hline $3^{\text {rd } \text { month }}$ & $4.52 \pm 3.61 *$ & $3.28 \pm 2.15^{*}$ & 0.068 \\
\hline
\end{tabular}

* $p<0.05$, when compared with the value before the block; * $p<0.05$, when compared between groups. GON: greater occipital nerve block; PGON: prophylaxis and greater occipital nerve block; SD: standard deviation.

Table 3. Comparison of the headache duration (hour; mean $\pm \mathrm{SD}$ )

\begin{tabular}{|l|c|c|c|}
\hline & $\begin{array}{c}\text { Group GON } \\
(\mathbf{n = 5 3 )}\end{array}$ & $\begin{array}{c}\text { Group PGON } \\
(\mathbf{n = 2 5})\end{array}$ & $\mathbf{p}$ \\
\hline Before block & $18.51 \pm 9.43$ & $15.20 \pm 9.16$ & 0.147 \\
\hline Week I & $11.62 \pm 11.46 *$ & $5.52 \pm 5.54 * \boldsymbol{q}$ & 0.004 \\
\hline Week 2 & $11.49 \pm 12.27 *$ & $5.84 \pm 5.64 * \boldsymbol{q}$ & 0.011 \\
\hline Week 3 & $10.21 \pm 8.92 *$ & $7.08 \pm 7.49 *$ & 0.123 \\
\hline Week 4 & $9.60 \pm 8.06 *$ & $4.36 \pm 3.99 * \boldsymbol{q}$ & 0.001 \\
\hline $2^{\text {nd }}$ month & $10.37 \pm 7.72 *$ & $7.16 \pm 4.40 *$ & 0.060 \\
\hline $3^{\text {rd }}$ month & $8.02 \pm 5.58 *$ & $7.20 \pm 4.16 *$ & 0.526 \\
\hline
\end{tabular}

${ }^{*} p<0.05$, when compared with the value before the block in both groups; $\pi_{p}<0.05$, when compared between groups. GON: greater occipital nerve block; PGON: prophylaxis and greater occipital nerve block; SD: standard deviation.

Table 4. Comparison of the headache severity (VAS; mean $\pm \mathrm{SD}$ )

\begin{tabular}{|l|c|c|c|}
\hline & $\begin{array}{c}\text { Group GON } \\
(\mathbf{n = 5 3 )}\end{array}$ & $\begin{array}{c}\text { Group PGON } \\
(\mathbf{n = 2 5})\end{array}$ & $\mathbf{p}$ \\
\hline Before block & $8.26 \pm 1.32$ & $8.08 \pm 0.90$ & 0.476 \\
\hline Week I & $5.44 \pm 2.74 *$ & $5.20 \pm 2.82^{*}$ & 0.718 \\
\hline Week 2 & $5.06 \pm 3.21^{*}$ & $5.32 \pm 2.93 *$ & 0.733 \\
\hline Week 3 & $5.24 \pm 2.87 *$ & $5.20 \pm 2.48 *$ & 0.941 \\
\hline Week 4 & $4.75 \pm 2.96 *$ & $4.16 \pm 2.77 *$ & 0.392 \\
\hline $2^{\text {nd }}$ month & $5.68 \pm 2.52 *$ & $6.18 \pm 1.08 *$ & 0.228 \\
\hline $3^{\text {rd }}$ month & $5.16 \pm 2.64 *$ & $5.96 \pm 1.20 *$ & 0.077 \\
\hline
\end{tabular}

* $p<0.05$, when compared with the value before the block; $p>0.05$, when groups were compared. VAS: visual analogue scale; GON: greater occipital nerve block; PGON: prophylaxis and greater occipital nerve block SD: standard deviation. 
and duration in the prophylactic medication plus GON block group was from 8.08 to 5.96 and from I 5.20 to 7.20 , respectively.

These results demonstrated the efficacy of GON blocking with or without medical prophylaxis, performed at certain intervals with local anesthetic in the treatment of migraine. GON blocking is a simple, safe, and effective method, with mild side effects, and can be used in patients with inadequate pain relief in spite of medical prophylaxis, or in those who have not tolerated medical treatment.

Conflict of Interest: No conflict of interest was declared by the authors.

Financial Disclosure: The authors declared that this study has received no financial support.

\section{REFERENCES}

I. Ertas M, Baykan B, Orhan EK, Zarifoglu M, Karli N, Saip S, Onal AE, Siva A. One-year prevalence and the impact of migraine and tension-type headache in Turkey: a nationwide home-based study in adults. J Headache Pain 2012; 13:147-I57. [CrossRef]

2. Mathew NT, Tfelt- Hansen P. General and pharmacologic approach to migraine management. In: Olesen J, Goadsby PJ, Ramadan MN, Tfelt-Hansen P, Welch KMA. The Headaches Third Edition. Philadelphia. Lippincott Williams \& Wilkins 2000, 433-440.

3. Terzi T, Karakurum B, Üçler S, Inan LE, Tulunay C. Greater occipital nerve blockade in migraine, tension-type headache and cervicogenic headache. J Headache Pain 2002; 3:137-|41. [CrossRef]

4. Caputi CA, Firetto V. Therapeutic blockade of the greater occipital and supraorbital in migraine patients. Headache 1997; 37:174- 179. [CrossRef]

5. Ashkenazi A, Young WB. The effects of greater occipital nerve block and trigger point injection on brush allodynia and pain in migraine. Headache 2005; 45:350-354. [CrossRef]

6. Afridi SK, Shields KG, Bhola R, Goadsby PJ. Greater occipital nerve injection in primary headache syndromes- prolonged effects from a single injection. Pain 2006; 122: 126- 129. [CrossRef]

7. Headache Classification Committee of the International Headache Society. The International Classification of Headache Disorders, 2nd Edition. Cephalalgia 2004; 24(Suppl I): I I 60.

8. Tobin JA, Flitman SS. Occipital nerve blocks: Effect of symptomatic medication: overuse and headache type on failure rate. Headache 2009; 49:1479| 485. [CrossRef]

9. Ashkenazi A, Matro R, Shaw JW, Abbas MA, Silberstein SD. Greater occipital nerve block using local anaesthetics alone or with triamcinolone for trans- formed migraine: a randomised comparative study. J Neurol Neurosurg Psychiatry 2008; 79:415-417. [CrossRef]

10. Young WB. The blocking greater occipital nerve: utility in headache management. Curr Pain Headache Rep 20 I0; 14:404-408. [CrossRef]

II. Bovim G, Sand I. Cervicogenic headache, migraine without aura and tension-type headache. Diagnostic blockade of the greater occipital and supraorbital nerves. Pain 1992; 51:43-48. [CrossRef]

12. Tobin J, Flitman S. Treatment of migraine with occipital nerve blocks using only corticosteroids. Headache 201 I; 51:155-159. [CrossRef]

13. Weibelt S, Andress-Rothrock D, King W, Rothrock J. Suboccipital nerve blocks for suppression of chronic migraine: Safety, efficacy, and predictors of outcome. Headache 20 I0; 50: I 04 I - 1044. [CrossRef]

14. Young WB, Mateos V, Ashkenazi A. Occipital nerve block rapidly eliminates allodynia far from the site headache: a case report. Cephalalgia 2004; 24:906907. [CrossRef]

15. Ashkenazi A, Young WB. The effects of greater occipital nerve block and trigger point injection on brush allodynia and pain in migraine. Headache 2005 45:350-354. [CrossRef]

16. Blumenfeld A, Ashkenazi A, Grosberg B, Napchan U, Narouze S, Nett B, DePalma T, Rosenthal B, Tepper S, Lipton RB. Patterns of Use of Peripheral Nerve Blocks and Trigger Point Injections Among Headache Practitioners in the USA: Results of the American Headache Society Interventional Procedure Survey (AHS-IPS). Headache 20 I 0; 50:937-942. [CrossRef]

17. Saracco MG, Valfre W, Cavallini M, Aguggia M. Greater occipital nerve block in chronic migraine. Neurol Sci 2010; 31 (Suppl I): 179-180. [CrossRef]

18. Gawel MJ, Rothbart PJ: Occipital nerve block in the management of headache and cervical pain. Cephalalgia 1992; 12:9-13. [CrossRef]

19. Inan N, Ceyhan A, Inan L, Kavaklioğlu O, Alptekin A, Unal N. C2/C3 nerve blocks and greater occipital nerve block in cervicogenic headache treatment. Funct Neurol 200 I; 16:239-243.

20. Karadaş Ö, Inan LE, Ulaş Ü, Odabaşı Z. Efficacy of lidocaine application on anxiety and depression and its curative effect on patients with chronic tension-type headache. Eur Neurol 20 I3; 70:95- I0I. [CrossRef]

2I. Karadaş Ö, Gül HL, Inan LE. Lidocain injection of pericranial myofascial trigger points in the treatment of frequent episodic tension-type headache. J Headache Pain 20|3; |4:44-5|. [CrossRef]

22. Afridi SK, Shields KG, Bhola R, Goadsby PJ. Greater occipital nerve injection in primary headache syndromes-prolonged effects from a single injection. Pain 2006; 122: I26-129. [CrossRef]

23. Goadsby PJ, Lipton RB, Ferrari MD. Migraine-current understanding and treatment. N Engl J Med 2002; 346:257-270. [CrossRef] 